$\mathrm{e}^{+} \mathrm{e}^{-}$Collisions from Phi to Psi 2013 (PHIPSI2013)

International Journal of Modern Physics: Conference Series

Vol. 35 (2014) 1460427 (4 pages)

(C) The Author

DOI: $10.1142 / \mathrm{S} 201019451460427 \mathrm{X}$

\title{
NUCLEON FORM FACTORS PROGRAM WITH SBS AT JLAB
}

\author{
BOGDAN WOJTSEKHOWSKI \\ Thomas Jefferson National Accelerator Facility \\ Newport News, Virginia 23606 USA \\ Published 18 December 2014
}

\begin{abstract}
The physics of the nucleon form factors is the basic part of the Jefferson Laboratory program. We review the achievements of the $6-\mathrm{GeV}$ era and the program with the 12 $\mathrm{GeV}$ beam with the SBS spectrometer in Hall A, with a focus on the nucleon ground state properties.
\end{abstract}

Keywords: Nucleon; form factors; flavor decomposition.

PACS Numbers: 14.20.Dh, 13.40.Gp

\section{The high $Q^{2}$ nucleon form factor experiments}

The nucleon structure investigation using high energy electron scattering has been a successful field where many discoveries have been made since the 1956 observation of the proton form factor. ${ }^{1}$ To a large extent, this success has been due to the dominance of the one-photon exchange mechanism of electron scattering, which allows reliable interpretation of the experimental data. ${ }^{2}$ By the early 90 s, the form factor data sets for the proton and the neutron were found to be mainly proportional to the one form factor, $F_{\text {Dipole }}=\left(1+Q^{2} / 0.71\left[\mathrm{GeV}^{2}\right]\right)^{-2}$ for all four: magnetic and electric for the proton, and magnetic and electric for the neutron. ${ }^{3}$

The most decisive studies of the partonic structure of nucleon could be performed when the dominant part of the wave function is a 3-quark Fock state. This requires large momentum transfer, $Q^{2}>1 \mathrm{GeV}^{2}$, when the contribution of the pion cloud is suppressed. The SLAC experimental data ${ }^{4}$ on the proton Dirac form factor $F_{1}^{p}$ have been found to be in fair agreement with a scaling prediction ${ }^{5}$ based on perturbative QCD: $F_{1}^{p} \propto Q^{-4}$, where $Q^{2}$ is the negative four-momentum transfer squared.

The experimental results ${ }^{6}$ from Jefferson Laboratory (JLab) for the ratio of the proton Pauli form factor $F_{2}^{p}$ and the Dirac form factor $F_{1}^{p}$ have been found to be in disagreement with the scaling law $F_{2}^{p} / F_{1}^{p} \propto 1 / Q^{2}$ suggested in Ref. 5 .

This is an Open Access article published by World Scientific Publishing Company. It is distributed under the terms of the Creative Commons Attribution 3.0 (CC-BY) License. Further distribution of this work is permitted, provided the original work is properly cited. 
A JLab high precision experiment made use of the double polarization method, which was first proposed in Ref. 7. This method is less sensitive to the two-photon exchange contribution and, due to the interference nature of the double polarization asymmetry, has large sensitivity to the small electric form factor. The data for $\mu_{p} G_{\mathrm{E}}^{p} / G_{\mathrm{M}}^{p}$ shown in Fig. 1(left) present an amazing drop of $G_{\mathrm{E}}^{p}$, which also means that $F_{1}^{p}$ and $Q^{2} \times F_{2}^{p}$ for the proton have different $Q^{2}$ dependencies.

The measurement of the proton to the neutron cross section ratio in the quasielastic knockout from the deuteron was used in JLab's precision measurement of the neutron magnetic form factor. ${ }^{8}$ With the recent JLab experiment on the neutron electric form factor, ${ }^{9}$ the data on all four nucleon form factors have become available in the $Q^{2}$ region of 3-quark dominance. Analysis of the flavor contributions to the nucleon form factors using the data was performed. ${ }^{10}$ The flavor decomposition allowed us to make two new observations:

- The contributions of the up quarks and down quark to the magnetic and electric form factors of the proton all have different $Q^{2}$ dependencies.

- The contribution of the down quark to the $F_{1}^{p}$ form factor at $Q^{2}=3.4 \mathrm{GeV}^{2}$ is three times less than the contribution of the up quarks (corrected for the number of quarks and their charge).

The second observation suggests that the probability of proton survival after the absorption of a massive virtual photon is much higher when the photon interacts with an up quark, which is doubly represented in the proton. This may be interpreted as an indication of an important role of the up-up correlation. At high $Q^{2}$ a correlation usually enhances the high momentum component and the interaction cross section. The relatively weak down quark contribution to the $F_{1}^{p}$ indicates a suppression of the up-down correlation or a mutual cancellation of different types of up-down correlations. The QCD-based calculations of the nucleon form factors in the Dyson-Schwinger Equations approach ${ }^{11}$ revealed a key role of the diquark in high $Q^{2}$ electron-nucleon elastic scattering.

\section{Future experiments in Hall A with SBS}

Accurate measurement of the FFs at large $Q^{2}$ will be possible during the next few years at Jefferson Lab, where the $12-\mathrm{GeV}$ energy upgrade is almost completed. ${ }^{12}$ In 2007 we proposed a configuration of a large acceptance spectrometer at a small angle to the beam. ${ }^{13}$ This large luminosity moderate acceptance spectrometer, SBS, become a key component of the form factor program in Hall A at JLab. The program includes the measurements of three ratios: the proton electric form factor to the proton magnetic form factor, ${ }^{14}$ the neutron electric form factor to the proton magnetic form factor, ${ }^{15}$ and the neutron electric form factor to the neutron magnetic form factor. ${ }^{16}$ For absolute normalization of the form factor values, the precision measurement of the proton magnetic form factor will also be performed in Hall A. ${ }^{17}$ A summary of experimental parameters is shown in Table 1. 

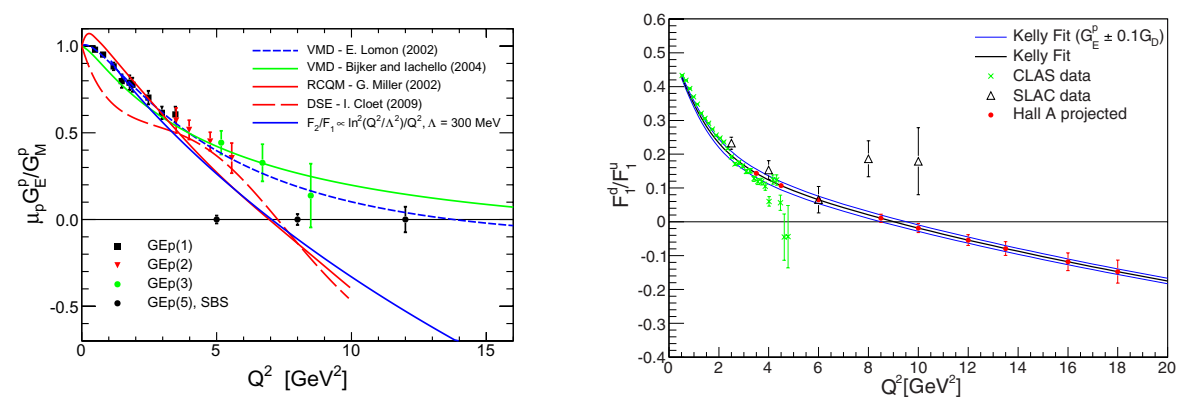

Fig. 1. Left: Existing data and projected data accuracy for the ratio of the $\mu_{p} G_{\mathrm{E}}^{p} / G_{\mathrm{M}}^{p}$. Right: Ratio of the up and down quark contributions to the proton form factor $F_{1}^{p}$.

Table 1. Future measurements of the FFs in Hall A at JLab (approved experiments). Projected range of $Q^{2}$ and accuracy relatively the dipole $\mathrm{FF}$ at maximum value of $Q^{2}$.

\begin{tabular}{cccl}
\hline Form factor & Reference & $Q^{2}$ range, $\mathrm{GeV}^{2}$ & $\Delta G / F_{\text {Dipole }}$ (stat/syst) at $\max Q^{2}$ \\
\hline$G_{\mathrm{E}}^{p}$ & 14 & $5-12$ & $0.08 / 0.02$ \\
$G_{\mathrm{M}}^{p}$ & 17 & $4.8-14.0$ & $0.01 / 0.02$ \\
$G_{\mathrm{E}}^{n}$ & 16 & $1.5-10.2$ & $0.09 / 0.03$ \\
$G_{\mathrm{M}}^{n}$ & 15 & $3.5-13.5$ & $0.06 / 0.03$ \\
\hline
\end{tabular}

\section{Flavor decomposition of the form factor $F_{1}^{p}$ and GPDs at very large $Q^{2}$}

At $Q^{2}$ above $10 \mathrm{GeV}^{2}$, measurement of the electric form factors, especially the $G_{\mathrm{E}}^{n}$, becomes difficult. However, due to the large value of $Q^{2} / 4 M_{N}^{2}$, the $F_{1}$ could be obtained with a relatively small uncertainty just from the value of the magnetic form factor:

$$
F_{1}=\left(G_{E}+Q^{2} / 4 M_{N}^{2} \times G_{M}\right) /\left(1+Q^{2} / 4 M_{N}^{2}\right)
$$

The flavor decomposition of $F_{1}$ also could be accomplished accurately. Fig. 1(right) shows projected data points and a systematic error corridor for assumed uncertainty in $G_{\mathrm{E}}^{p}$ of \pm 0.1 . Here we used the Kelly fit form factors for illustration purposes. We would like to note that the ratio $F_{1}^{d} / F_{1}^{u}$ could potentially cross the zero line, which would require a significant change of GPDs parametrization because the currently used form does not allow negative values of GPDs, see e.g. the reference. ${ }^{18}$

\section{Acknowledgments}

The author would like to thank the organizing committee of the "International Workshop on e+e- collisions from Phi to Psi" for the invitation to present a talk. This work was supported in part by the U. S. National Science Foundation, and by Department of Energy (DOE) contract number DE-AC05-06OR23177, under which 
the Jefferson Science Associates operates the Thomas Jefferson National Accelerator Facility.

\section{References}

1. E.E. Chambers and R. Hofstadter, Phys. Rev. 103, 1454 (1956).

2. M.N. Rosenbluth, Phys. Rev. 79, 615 (1950).

3. P. Bosted, Phys. Rev. C 51, 409 (1995).

4. R.G. Arnold et al., Phys. Rev. Lett. 57, 174 (1986); A.F. Sill et al., Phys. Rev. D 48, 29 (1993).

5. G.P. Lepage and S.J. Brodsky, Phys. Rev. Lett. 43, 545 (1979); 43, 1625(E) (1979).

6. M.K. Jones et al., Phys. Rev. Lett. 84, 1398 (2000); O. Gayou et al., Phys. Rev. C 64, 038202 (2001); O. Gayou et al., Phys. Rev. Lett. 88, 092301 (2002); V. Punjiabi et al., Phys. Rev. C 71 (2005) 055202; A. Puckett et al., Phys. Rev. Lett. 104, 242301 (2010); A. Puckett et al., Phys. Rev. C 85 (2012) 045203.

7. A.I. Akhiezer, L.N. Rosenzweig and I.M. Shmushkevich, Sov. Phys. JETP 6, 588 (1958); A.I. Akhiezer and M. P. Rekalo. Sov. J. Part. Nucl., 3:277, 1974; R.G. Arnold, C.E. Carlson, and F. Gross, Phys. Rev. C 23, 363 (1981).

8. J. Lachniet et al., Phys. Rev. Lett. 102, 192001 (2009).

9. S. Riordan et al., Phys. Rev. Lett. 105, 262302 (2010).

10. G. Cates et al., Phys. Rev. Lett. 106, 252003 (2011).

11. C.D. Roberts et al., Eur. Phys. J. ST 140, 53 (2007); I.C. Cloët et al., Few-Body Systems 46, 1(2009).

12. Thomas Jefferson National Accelerator Laboratory, https://www.jlab.org

13. Super Bigbite Spectrometer, http://hallaweb.jlab.org/12GeV/SuperBigBite/

14. JLab experiment E12-07-109, E. Brash, E. Cisbani, M. Jones, M. Khandaker, L. Pentchev, C.F. Perdrisat, V. Punjabi, and B. Wojtsekhowski et al., http://www.jlab.org/exp_prog/PACpage/PAC32/talks/wojtsekhowski.pdf

15. JLab experiment E12-09-019, J. Annand, G. Gilman, B. Quinn and B. Wojtsekhowski et al., http://www.jlab.org/exp_prog/PACpage/PAC34/talks/09-019.pdf

16. JLab experiment E12-09-016, G. Cates, S. Riordan, and B. Wojtsekhowski et al., http://www.jlab.org/exp_prog/PACpage/PAC34/talks/09-016.pdf

17. JLab experiment E12-07-108, J. Arrington, E. Christy, S. Gilad, B. Moffit, V. Sulkosky and B. Wojtsekhowski et al., http://www.jlab.org/exp_ prog/PACpage/PAC32/talks/PR12-07-108.pdf

18. M. Diehl and P. Kroll, Eur.Phys.J. C73 (2013) 2397; arXiv:1302.4604. 